\title{
Idiopathic Fatal Pancytopenia: A Case Report
}

\author{
Helen Shaji John Cecily ${ }^{1}$, Retnam Golda Sahayarani ${ }^{2}$
}

\begin{abstract}
Idiopathic fatal pancytopenia is a rare disease with a grave prognosis. Pancytopenia is defined as reduction in all three types of blood cells, such as erythrocytes, leukocytes, and thrombocytes. Erythrocytes transport oxygen to parts of our body; leukocytes play a major role in our body by fighting against infection and destroying pathogens; thrombocytes act as primary agents to stop bleeding at the site of injury. Pancytopenia predisposes to increase the chances of infection and bleeding tendencies. ${ }^{1-3} \mathrm{~A} 41$-year-old female client got admitted in a hospital with the signs and symptoms of giddiness, bleeding per vagina for 20 days, breathlessness on exertion, generalized body weakness, vomiting, and pain abdomen. There were no abnormal findings on physical and systemic examination. Complete blood count (CBC) findings showed decreased number of all three kinds of cells, whereas other hematological parameters were normal. Stool examination revealed presence of occult blood with no abnormal findings on ultrasonography (USG). On bone marrow examination, hypercellular bone marrow was seen with reduction of all three blood cells. Patient was admitted in hospital for 20 days. The cause of pancytopenia remained unexplained. Following administration of blood and blood products, inotropes and hematinic therapy led to the recovery of the blood cell components to normal level. Bone marrow examination later became normal. Idiopathic fatal pancytopenia is a rare condition based on clinical and pathological features.

Keywords: Bleeding, Bone marrow, Case report, Complete blood count, Idiopathic fatal pancytopenia.

Pondicherry Journal of Nursing (2020): 10.5005/jp-journals-10084-12161
\end{abstract}

\section{BACKGROUND}

Pancytopenia is defined as reduction in all three types of blood cells, such as erythrocytes, leukocytes, and thrombocytes (Fig. 1). Major symptoms of pancytopenia will be seen only during stressful conditions. A timely intervention is required to avoid complications. The appropriate clinical history is required to treat the patient of pancytopenia. Without treatment, idiopathic fatal pancytopenia can become life-threatening. ${ }^{4-6}$

\section{Case Description}

\section{Patient History}

A 41-year-old woman, not a known case of diabetes mellitus and hypertension came to the hospital with the chief complaints of giddiness, bleeding per vagina 20 days, breathlessness on excretion, generalized body weakness, vomiting, and pain abdomen. Consulted a physician in a private hospital and initially she was diagnosed to have anemia and she was treated for the same but no positive results. Moreover, no abnormalities were identified in endoscopy. Furthermore, she consulted a hematologist and bone marrow examination was performed. Following investigations were performed, such as fecal occult blood test, liver and renal function tests, lipid profile, complete blood count (CBC) coagulation test, viral marker profile, and ultrasonography (USG) of the abdomen. Based on the results, the patient was diagnosed as idiopathic fatal pancytopenia.

\section{Results of the Physical Exam}

On examination, the patient appeared icterus, mild pallor and absence of clubbing, lymph adenopathy, and edema of the foot. No abnormalities were detected in systemic examination. While checking her vital signs, heart rate was 78 beats $/ \mathrm{min}$, respiratory rate was 14 breaths $/ \mathrm{min}$, temperature was $97.6^{\circ} \mathrm{F}$ $\left(36.4^{\circ} \mathrm{C}\right)$, blood pressure was $120 / 70 \mathrm{~mm} \mathrm{Hg}$, and oxygen saturation was $97 \%$.
1,2Department of Medical Surgical Nursing, Shri Sathya Sai College of Nursing, Chennai, Tamil Nadu, India

Corresponding Author: Helen Shaji John Cecily, Department of Medical Surgical Nursing, Shri Sathya Sai College of Nursing, Chennai, Tamil Nadu, India, Phone: +91 9383880999, e-mail: helenshaji@gmail. com

How to cite this article: Cecily HSJ, Sahayarani RG. Idiopathic Fatal Pancytopenia: A Case Report. Pon J Nurs 2020;13(4):86-88.

Source of support: Nil

Conflict of interest: None

\section{Laboratory and Other Results}

Complete blood count showed decreased number of all three cell components: RBC count-3.6 UL, total leukocyte count (TLC) - 2300 cells $/ \mathrm{mm}^{3}$, platelet count-70,000 cells $/ \mathrm{mm}^{3}$, with hemoglobin $(\mathrm{Hb})-5.3 \mathrm{~g} / \mathrm{dL}$, mean corpuscular hemoglobin $(\mathrm{MCH})-28.9 \mathrm{pg}$, mean corpuscular volume $(\mathrm{MCV})-89.1 \mathrm{fL}$, mean corpuscular hemoglobin concentration ( $\mathrm{MCHC}$ - $-333 \mathrm{~g} / \mathrm{dL}$, reticulocyte count was $0.8 \%$. Fecal test for occult blood showed positive. Other tests, such as lipid profile, liver and renal function tests, and urine routine tests, showed normal values. Blood coagulation profile was within normal range. Viral markers, such as hepatitis $B$ surface antigen (HBsAg), hepatitis A virus (HAV), hepatitis C virus (HCV), HIV, and Ebstein-Barr virus (EBV), and dengue were negative. Abdominal ultrasound test revealed splenomegaly; however, CT scan and endoscopy showed no evidence of abnormalities. On bone marrow examination, hypercellular bone marrow was seen with depression of all three blood cells (Fig. 2). Bone marrow biopsy revealed normal size, shape, and distribution of all three cellular components.

\section{Primary Outcomes of Treatment}

In the beginning, patient was started with symptomatic management, such as hematinic therapy for anemia; however, no significant improvement was observed. Patient's Hb decreased to 

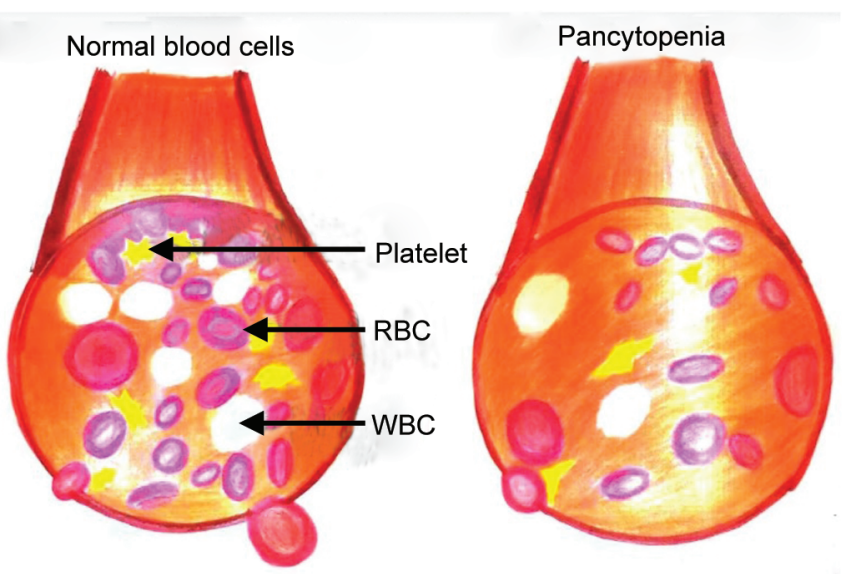

Fig. 1: Difference between normal blood cells and pancytopenic blood cells

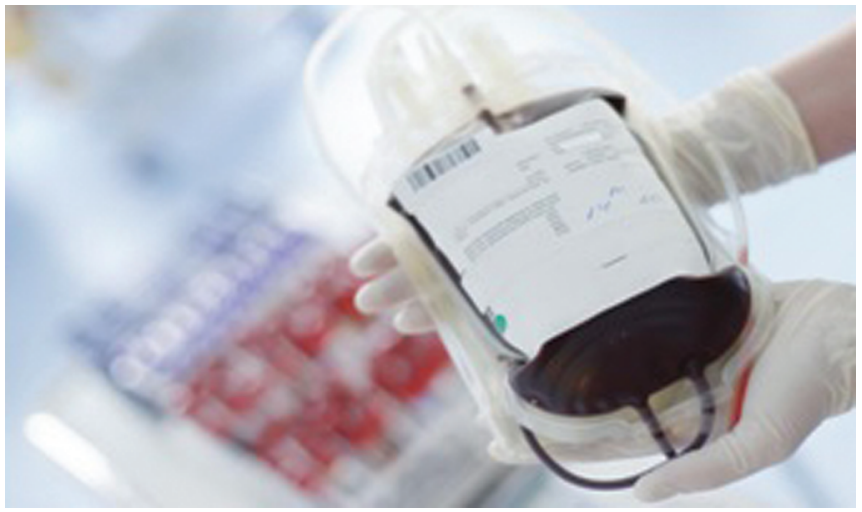

Fig. 3: Transfusion of blood for pancytopenia patient

$4.9 \mathrm{~g} / \mathrm{dL}$; hence, she was transfused with two units of packed RBC (PRBC). Prothrombin time (PT), international normalized ratio (INR), activated partial thromboplastin time (APTT), bleeding time, and coagulation profile were repeated and were within normal range (Fig. 3). On the 3rd day of admission, she developed respiratory distress, and tachycardia along with persistent hypotension. Immediately resuscitative measures were initiated, such as fluid administration and blood transfusion, but the patient's vitals continued to remain unstable. Hence, as per the institutional protocols, inotropes, massive blood transfusion, and fresh frozen plasma (FFP) were given. Patient's vitals and hematological parameters started getting stable. The patient's blood counts improved and came to normal limits by week 4 after treatment. She did not experience any infections, complications, or bleeding in the later period of stay at the hospital.

\section{Discussion}

Pancytopenia can be detected when an abnormal clinical finding, such as easy fatigability, infection, or bleeding, and is subjected for further evaluation or sometimes, can even be spotted incidentally during routine checkups. It is a presenting feature in many diseases, and the severity and outcome depend on the etiology of pancytopenia. Hematological and non-hematological disorders can involve the bone marrow predominantly or tangentially, and lead to the clinical features of pancytopenia., ${ }^{78}$

In the present case, initially the patient had severe anemia, vaginal bleeding, breathlessness, and pain abdomen with RBC

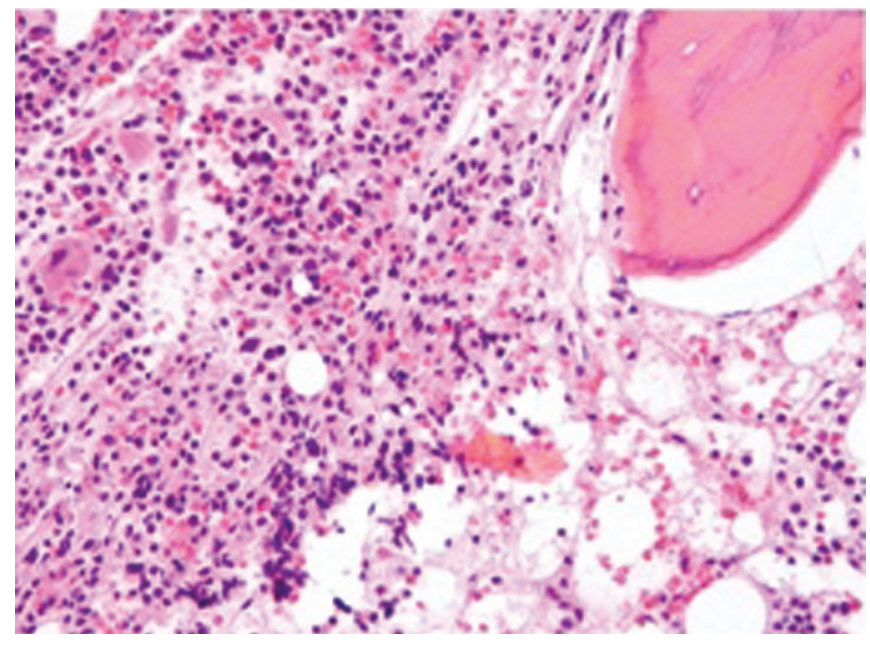

Fig. 2: Histological features demonstrating hypercellular bone marrow

count-3.6 UL, TLC -2300 cells $/ \mathrm{mm}^{3}$, platelet count $-70,000$ cells/ $\mathrm{mm}^{3}$, with Hb-5.3 g/dL, MCH-28.9 pg, MCV-89.1 fL, MCHC-333 $\mathrm{g} / \mathrm{dL}$, reticulocyte count was $0.8 \%$. After the intensive course of treatment, gradually she started showing improvement in her general health and the hematological parameters, such as $\mathrm{Hb}-9$ $\mathrm{g} / \mathrm{dL}, \mathrm{RBC}$ count $-4.2 \mathrm{UL}, \mathrm{TLC}-4500$ cells $/ \mathrm{mm}^{3}$, platelet count$1,20,000$ cells $/ \mathrm{mm}^{3}, \mathrm{MCH}-30.2 \mathrm{pg}, \mathrm{MCV}-92.2 \mathrm{fL}, \mathrm{MCHC}-352 \mathrm{~g} /$ $\mathrm{dL}$, reticulocyte count was $1.6 \%$ within normal limits. Bone marrow examination results turn to normal. This shows that early diagnosis and timely management saved the life of the patient without leaving any complications. Hence, early diagnosis and timely intervention is essential to improve the quality of life of patient with pancytopenia.

\section{Nursing Care Rendered to the Patient}

Nursing care involved a major role caring for patients with idiopathic fatal pancytopenia. The patient was prepared both physically, mentally, and psychologically before the treatments. Informed consent was obtained; elucidated the nature of treatments, benefits, and their complications.

- Provided a calm, quiet, and stable environment.

- Maintained complete bed rest to the patient.

- Observed the patient for any signs of infection, such as increased body temperature, chills, pain, and fatigue.

- Monitored $\mathrm{Hb}$, hematocrit, RBC counts, and reticulocyte counts regularly.

- Monitored vital signs every 4th hourly, assessed for capillary refill, color of skin, mucous membranes, and nail beds.

- Nursing care was provided before, during, and after bone marrow biopsy.

- Explained the procedure thoroughly to the patient and mild sedative was administered 1 hour prior to the test.

- Oriented the patient that the test usually lasts for 5-10 minutes and more than one site may be used for collecting specimen.

- Allergic test was performed to rule out the hypersensitivity to the local anesthetic.

- Explained the patient about various sites used for bone marrow biopsy, such as sternum, anterior or posterior iliac crest, vertebra, spinous process, ribs, or tibia.

- Cleaned the biopsy site and applied a sterile dressing. 
- The marrow slides were prepared, and sent to the lab for investigation to rule out the cause.

- Periodically checked the patient's vital signs and observed the biopsy site for signs and symptoms of infection.

- Nursing care was given during blood transfusion.

- Administered blood transfusion as ordered, checked for allergic reaction, circulatory overload or hemolytic reaction.

- Before starting each unit of blood transfusion, the patient's baseline vital signs were recorded and were repeated every 15 and 30 minutes.

- Monitored platelet count periodically and instructed the client/family members about the precautions to be taken to avoid bleeding.

- Closely monitored oxygen saturation and administer oxygen. $\mathrm{SpO}_{2}$ is $<94 \%$, delivered oxygen via nasal cannula at $2 \mathrm{~L} / \mathrm{min}$.

- Iron supplements were regularly administered an hour before meals for maximum absorption; advised the patient to take the supplement with meals if gastric distress occurs and switch over to between-meals schedule once the symptoms subsided.

- Psychological support and diversional therapy were given.

- Advised the client to consume iron and vitamin C rich foods to compensate the blood loss and prevent infection.

- Stressed the importance of personal hygiene, oral care, and perineal care. Advised to use a soft toothbrush and soft wash cloth to prevent skin and gums bleeding.

- Assessed the skin for bruises and petechiae for the patient.

- Instructed to avoid gatherings and crowded places and avoid contact with sick or diseased.

- Reinforced the importance of adhering to treatment regimen and keeping follow-up appointments.

\section{Conclusion}

Pancytopenia is a rare hematological problem came across in clinical practice. Patient's age, gender, physical examination, history, laboratory test results, and USG findings provide important indicators in the diagnosis of pancytopenia. Etiological causes of pancytopenia and their rates may differ in each cases from same and different countries. Henceforth, most of the etiological causes of pancytopenia were associated with non-hematological diseases and were diagnosed with laboratory tests without the need of a bone marrow examination. Awareness about the various causes, which is generally region specific and about the specific laboratory findings associated with each one and also to avoid unnecessary invasive procedures when not indicated. Reverse barrier isolation is one of the best cost-effective nursing interventions in the care of pancytopenia. ${ }^{9,10}$ Personal hygiene, meticulous skin care, great dental consideration, and rectal cleanliness are significant. Legitimate handwashing by medical and health professional routinely before looking at any patient of pancytopenia is a basic methodology for preventing infection. If pancytopenia is left untreated, it can have potential life-threatening complications which may include severe bleeding and infections.

\section{References}

1. Javalgi AP, Dombale VD. Clinico-hematological analysis of pancytopenia: a bone marrow study. National J Lab Med 2013;2(4):1217. DOI: NJLM/2013/6782:1995.

2. Manzoor F, Karandikar MN, Nimbargi RC. Pancytopenia: a clinicohematologic study. Med J Dr DY Patil University 7(1):25-28. DOI: 10.4103/0975-2870.122763.

3. Sandhya V. Bone marrow pathology in pancytopenia. Int J Sci Res 2016;5(5):323-325. ISSN No. 2277-8179, IF: 3.508, IC Value: 69.48.

4. Negotia P, Saxena RS, Jagat RS, Dubey TN. Clinical etiological evaluation of pancytopenia. Int J Med Res Rev 2017;5(11):927-932. DOI: 10.17511/ijmrr.2017.i11.02. Print ISSN: 2321-127X, Online ISSN: 2320-8686.

5. Tekwani D, Bawa S, Joshi R, Joshi SR. Clinico-haematological analysis of pancytopenia in adults. J Med Sci Clin Res 2017;5(3):19020-19026. DOI: $10.18535 / \mathrm{jmscr} / \mathrm{v} 5 \mathrm{i} 3.115$.

6. Madhuchanda K. Pancytopenia. J Indian Acad Clin Med 2002;3(1):2934.

7. Jha A, Sayami G, Adhikari RC, Panta AD, Jha R. Bone marrow examination in cases of pancytopenia. J Nepal Med Assoc 2008;47(169):12-17. DOI: 10.31729/jnma.209.

8. Pathak R, Jha A, Sayami G. Evaluation of bone marrow in patients with pancytopenia. J Pathol Nepal 2012;2(4):265-271. DOI: 10.3126/ jpn.v2i4.6875.

9. Khodke K, Marwah S, Buxi G, Vadav RB, Chaturvedi NK. Bone marrow examination in cases of pancytopenia. J Academy Clin Med 2001;2(1):55-59.

10. Santra G, Das BK. A cross-sectional study of the clinical profile and aetiological spectrum of pancytopenia in a tertiary care center. Singapore Med J 2010;51(10):806-812. 